\title{
MAGNETO-OPTICAL MEDIUM FOR OPTICAL TAPE RECORDING
}

\author{
H. Tokumaru, K. Arai, S. Yoshimura, K.Takizawa, N. Uchida* and H. Yoshida* \\ NHK Science \& Technical Research Laboratories, 1-10-11 Kinuta, Setagaya, Tokyo 157, Japan \\ *Research Center, Mitsubishi Chemical Corp., Kamoshida, Aoba-ku, Yokohama 227, Japan
}

\begin{abstract}
The requirements for optical tapes in an optical tape recording system using a rotating optical head are presented. Along with the requirements, the best structure for a magneto-optical (MO) tape was studied. As a result, a tri-layer structure was adopted for easy production and the tri-layerd MO tapes were experimentally fabricated. Performances and problems of an MO tape were experimentally investigated using these samples. And, the following resulis were obtained which realized performances equal to an optical disk using a thin polyethylene terephthalate (PET) substrate. (1) The incidence of the laser is from the tri-layer side to avoid the birefringence of the PET substrate. (2) Surface roughness of the PET substrate is below $R a=7 \mathrm{~nm}$. (3) Heat-resistance of PET substrate makes recording or erasing operations in excess of ten thousand times possible. (4) MO tape (width: $1 / 2$ inch, thickness: $10 \mu \mathrm{m}$ ) was stable in terms of optical and magnetic properties under a tape tension of $0.5 \mathrm{kgw}$. (5) Sufficient tape tension is necessary to satisfy the requirement of flatness since MO tape has a large extent of curling.
\end{abstract}

KEYWORDS: MAGNETO-OPTICAL TAPE, OPTICAL TAPE RECORDING SYSTEM, PET, CURLING, BIREFRINGENCE, ROUGHNESS, HEAT-RESISTANCE

\section{INTRODUCTION}

An optical tape recording system is capable of providing a huge video library in a small space due to the feasibility of a compact cassette with an extremely large capacity exceeding 1 Tbytes. There are three types of tape media l.e. ROM, WO, and an erasable type. Among these, we have been studying an erasable magneto-optical ( MO) tape to be used in video libraries and future TV memories at broadcasting stations. And, stable recording on a thin polyethylene terephthalate (PET) substrate could be accomplished [1].

Until now, there have been two reports on the MO tape media. One is that the magnetic properties did not vary under practical tape tension using $\mathrm{TbFe}$ film deposited on a $25 \mu \mathrm{m}$-thick substrate [2]. The other one is that the carrier to noise ratio ( CNR ) equivalent to an MO disk was realized using the fabricated tape in the same way as a disk[3]. However, these are only partial reports on the performances and problems of the MO tape.

In this report, at first, we present the requirements for optical tapes in the optical tape recording system using the rotating optical head [4]. Next, performances of experimentally fabricated MO tapes are described, and their problems and feasibility are also discussed.

\section{REQUIREMENTS FOR OPTICAL TAPE}

The three major requirements for an optical tape in the optical tape recording system are as follows.

(1) Performances equal to an optical disk using a thin substrate

- Low surface roughness of the substrate

- Heat-resistance of the substrate

- Stability of optical and magnetic properties under tape tension and bending
- Long archival life

(2) Easy production of long tapes owing to simple structure

(3) Good matching with the recording system using the rotating optical head

- No factors such as curling to degrade the flatness of the tape.

Since the range of focusing operations is designed to be about $60 \mu \mathrm{mp}-\mathrm{p}$ ( $\mu \mathrm{m}$, peak to peak value ) in the optical tape recording system, the tape must be positioned below such flatness within the scanning area [5] and have a low axial acceleration below the value determined by the performance of the focusing actuator. The tension on the optical tape is effective in realizing the flatness. However, the tape tension is expected to be less than or equal to the tension of the magnetic tape, that is below $0.05 \mathrm{kgw}$ for a tape of a half inch width, in a videotape recorder (VTR) because of high reliability of the tape and low electric power consumption.

- Good tape running

\section{STRUCTURE OF MAGNETO-OPTICAL TAPE}

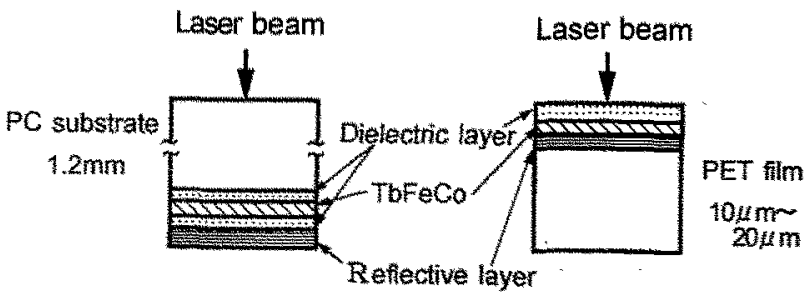

Magneto-optical disk

Magneto-optical tape

Fig.1. Structures of the MO disk and MO tape. 
The typical structure of an MO disk and the structure of an experimental MO tape are both shown in Fig. 1 An MO disk has a quadri-layered structure ( reflective/ dielectric/ $\mathrm{TbFeCo} /$ dielectric) deposited on a polycarbonate (PC) substrate $1.2 \mathrm{~mm}$ thick, in which a $\mathrm{TbFeCo}$ magnetic layer is sandwiched between two dielectric layers to prevent the oxidation of $\mathrm{TbFeCo}$ and enhance the Kerr rotation. An MO tape has similar layers deposited on a non rigid substrate. A PET substrate was employed because it had been used for magnetic tapes. We studied the best structure of an MO tape using a PET substrate.

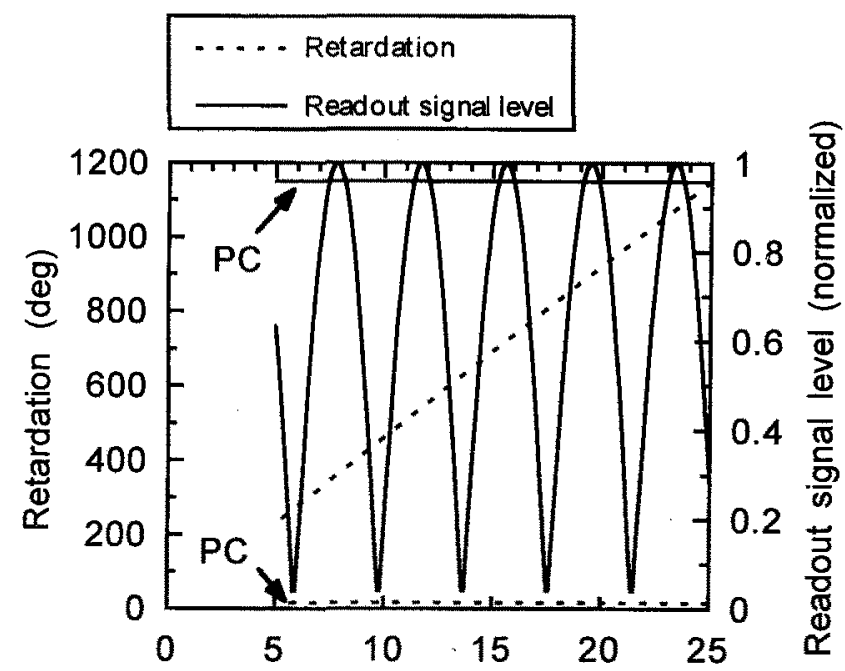

PET film thickness $(\mu \mathrm{m})$

Fig.2. Effect of birefringence of PET substrate.

A PET substrate has much larger birefringence compared with a PC substrate since a PET substrate has a bi-axial orientation. For example, in the case of vertical incidence of the laser light to a substrate, the difference between the maximum refractive index and the minimum one, that is described here as $\Delta n_{3}$ is about $15 \times 10^{-6}$ in a PC substrate and $\Delta n=50 \times 10^{-3}$ in a PET one.

Figure 2 shows the effect of the retardation, that is defined here as $\delta$ degrees, caused by $\Delta \mathrm{n}$. The retardation changes in proportion to the thickness of the PET substrate, and it causes the variation in amplitude of the readout signal as a cosine function $(\cos (\delta))$. Therefore, the level of the readout signal from an MO tape changes remarkably. The effect of the retardation in a PC substrate is also shown in Fig. 2 . There is little decrease in the readout signal level because of minimal retardation. Accordingly, it is necessary that the magnetic layer be directly illuminated to avoid the influence of the PET's large birefringence as shown in Fig.1.

A quadri-layered structure is usually employed for a disk. However, a simpler structure is better for an
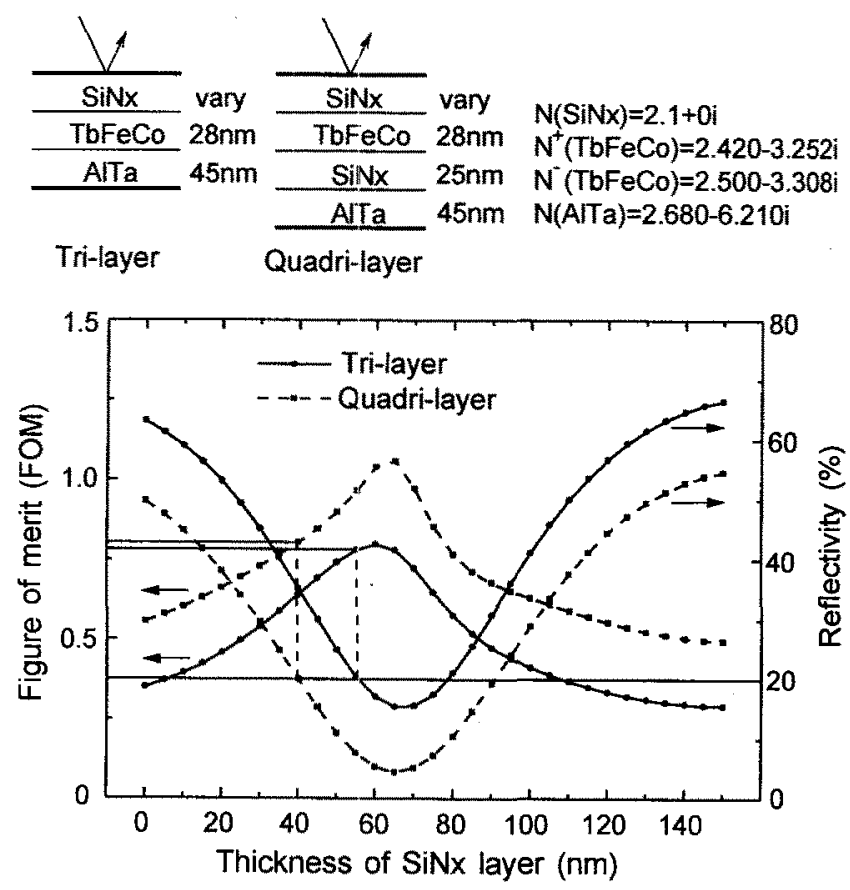

Fig.3. Comparison between tri-layer and quadrilayer.

MO tape due to its large deposition area. Calculated results on the reflectivity $(R)$ and FOM ( Figure Of Merit: $\theta_{k} \sqrt{R}$ ) of both structures, that are a quadrilayer and a tri-layer, are shown in Fig.3. Calculations were carried out by changing the thickness of the top dielectric layer. in the case of a quadri-layer structure, the thickness of the lower dielectric layer was fixed at $250 \mathrm{~nm}$.

The FOM of the quadri-layer is much larger than that of the tri-layer for all dielectric layer thicknesses. However, both FOM values at a reflectivity of $20 \%$, that is a practical value in terms of the servo stability and the quality of readout signal, are almost equal. This means that a tri-layer structure is better for an MO tape because of easy production. Accordingly, we fabricated the tri-layered MO tape media using the sputtering method shown in Table 1 in order to investigate the characteristics.

Table 1. Sputtering conditions of MO tape.

\begin{tabular}{|c|c|c|c|}
\hline MATERIAL & POWER & $\begin{array}{c}\text { Ar } \\
\text { PRESSURE }\end{array}$ & $\begin{array}{c}\text { DEPOSITION } \\
\text { RATE }\end{array}$ \\
\hline AITa & $750 \mathrm{~W}$ & $3.2 \mathrm{mTOrr}$ & $0.34 \mathrm{~nm} / \mathrm{s}$ \\
TbFeCo & $700 \mathrm{~W}$ & $2.9 \mathrm{mTOrr}$ & $0.31 \mathrm{~nm} / \mathrm{s}$ \\
SiNx & $525 \mathrm{~W}$ & $5.8 \mathrm{mTorr}$ & $0.13 \mathrm{~nm} / \mathrm{s}$ \\
\hline
\end{tabular}




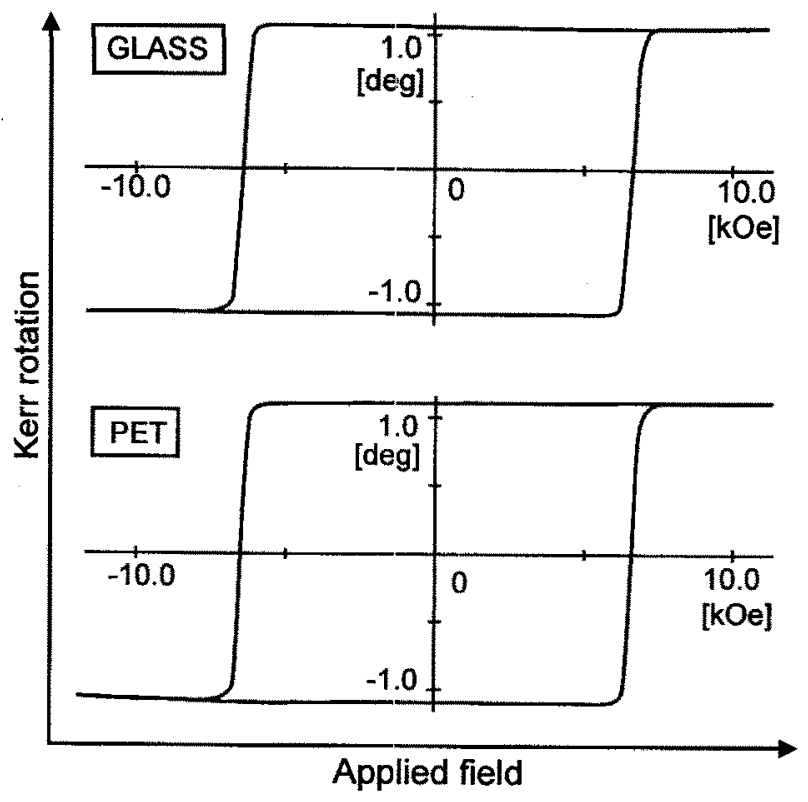

Fig.4. Kerr hysteresis loops.

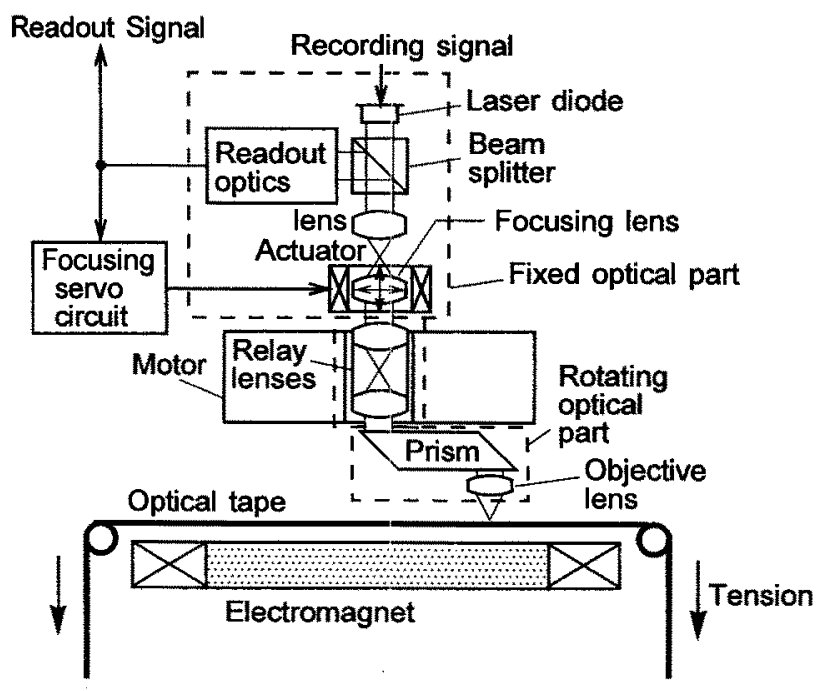

Rotation speed: $1000 \mathrm{rpm}$ Radius of rotation: $30 \mathrm{~mm}$ NA of objective lens: 0.5 Wavelength: $790 \mathrm{~nm}$

Fig.5. Experimental setup.

\section{CHARACTERISTICS OF EXPERIMENTAL TAPES}

\section{Surface roughness}

The MO medium on a thin PET substrate has several problems. One of them is that the surface roughness of a PET substrate is generally much larger than that of a PC substrate. Three layers (AITa: $45 \mathrm{~nm}$, TbFeCo: $28 \mathrm{~nm}$, SiNx: $550 \mathrm{~nm}$ ) were deposited on a glass substrate ( $1.2 \mathrm{~mm}$-thick disk, Ra: $2.4 \mathrm{~nm}$ ), a PC substrate ( $1.2 \mathrm{~mm}$-thick disk, Ra: $5.1 \mathrm{~nm}$ ) and three kinds of PET substrates ( width: $55 \mathrm{~mm}$, length: 600 $\mathrm{mm}$, thickness: $20 \mu \mathrm{m}$, Ra: $6.5 \mathrm{~nm}, 9.6 \mathrm{~nm}, 16.3 \mathrm{~nm}$ ) under the same conditions. The values of $\theta$ and $R$ of the PC disk were $1.0^{\circ}$ and $23 \%$ respectively. The surface roughness ( $\mathrm{Ra}$ ) was measured with a interferometric microscope (MAXIM-3D, Zygo corp.).

Figure 4 shows the measured Kerr hysteresis loops of the glass substrate and the PET substrate (Ra: $16.3 \mathrm{~nm}$ ). Since they are almost equal, there is no difference in magnetic properties in spite of differences in surface roughness. Recording and readout evaluations of the two disks and three kind of tapes were carried out using the experimental setup shown in Fig.5. An optical head is rotated with a precision of $0.05 \mu \mathrm{m}$ in a radial direction by a motor.

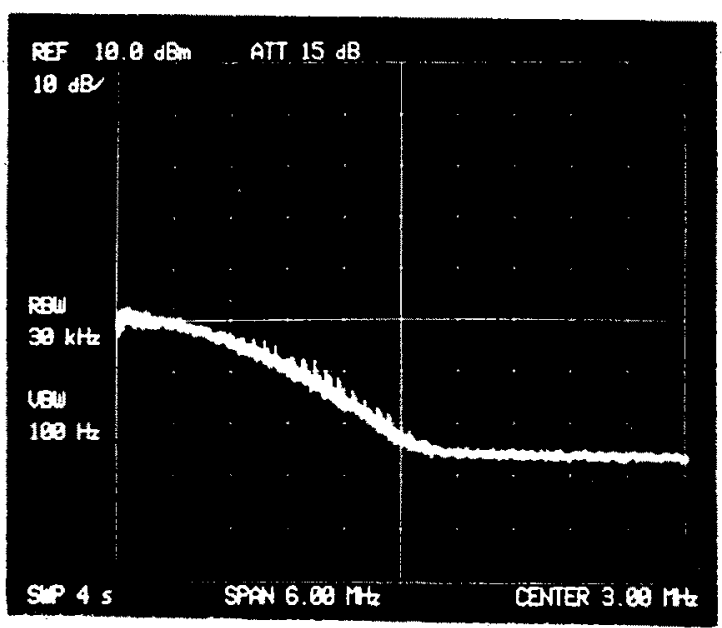

Fig.6. Readout spectrum after erasing the tape (Ra:16.3nm).

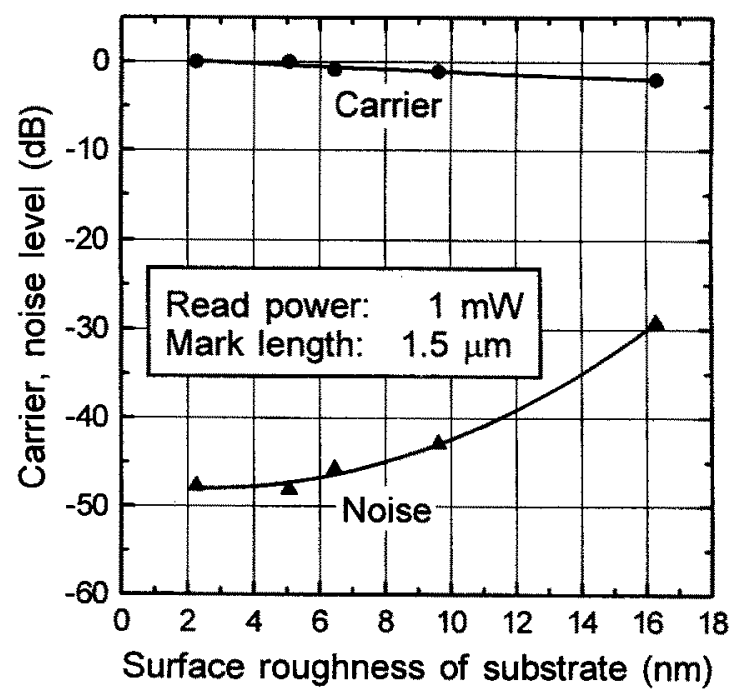

Fig.7. Carrier and noise vs surface roughness of medium. 
Each recording medium using a PET substrate was flattened along the two parallel rods, which were located at an interval of $140 \mathrm{~mm}$, by exerting the tape tension beyond $2 \mathrm{kgw}$. And, it was installed under the optical head for recording and readout evaluations.

The photograph of the readout spectrum after erasing the tape ( $\mathrm{Ra}: 16.3 \mathrm{~nm}$ ) is shown in Fig.6. Noise appeared owing to significant roughness of the surface. Figure 7 shows the relation between the PET' $s$ surface roughness and the readout carrier $(C)$ level and the noise $(\mathrm{N})$ level at mark length of $1.5 \mu \mathrm{m}$. N level increased remarkably in accordance with increments of surface roughness while the $C$ level remained almost constant. The CNR was almost the same value as a disk below surface roughness of $7 \mathrm{~nm}$. Therefore, the surface roughness of the PET substrate must be reduced to be equal to that of a disk substrate

\section{Heat-resistance of substrate}

The glass transition temperature ( $\mathrm{Tg}$ ) of a PET substrate is about $70^{\circ} \mathrm{C}$ and lower than that of a PC substrate by $70^{\circ} \mathrm{C}$. And, they have almost the same melting point ( $\mathrm{Tm}$ ) of about $250^{\circ} \mathrm{C}$. Therefore, the thermal damage to a PET substrate by repeated erasing and recording operations is a concern. We estimated the temperature rise in a PET substrate while recording a mark of $1.5 \mu \mathrm{m}$ by a difference calculus method under the conditions shown in Table 2.

Table 2. Conditions for calculation of temperature rise in PET film.

\begin{tabular}{|c|c|c|c|l|}
\hline MEDIUM & THICKNESS & $\begin{array}{c}\text { SPECIFIC } \\
\text { HEAT } \\
\left(\mathrm{pJ} / \mu \mathrm{m}^{3} \cdot{ }^{\circ} \mathrm{C}\right)\end{array}$ & $\begin{array}{c}\text { THERMAL } \\
\text { CONDUCTIVITY } \\
\left(\mathrm{pJ} / \mu \mathrm{m}^{3} \cdot{ }^{\circ} \mathrm{C} \cdot \mathrm{ns}\right)\end{array}$ & $\begin{array}{c}\text { REFRACTIVE } \\
\text { INDEX }\end{array}$ \\
\hline PET & 10 & 1.70 & 0.00075 & $1.50+\mathrm{j0}$ \\
$\mathrm{AITa}$ & 0.045 & 2.70 & 0.040 & $2.68-\mathrm{j} 6.21$ \\
TbFeCo & 0.028 & 3.20 & 0.020 & $2.50-\mathrm{j} 3.30$ \\
SiNx & 0.055 & 3.52 & 0.001 & $2.10+\mathrm{j} 0$ \\
AIR & 10 & 0.35 & 0.00025 & $1.00+\mathrm{j} 0$ \\
\hline
\end{tabular}

- Laser wavelength: $780 \mathrm{~nm}$ - Laser power: $4 \mathrm{~mW}$

- Mark length: 1.5 um $\quad$ Duty: $30 \%$

- Velocity: $3.14 \mathrm{~m} / \mathrm{s}$

- Room temperature: $25^{\circ} \mathrm{C}$

The phase changes of a PET substrate were not taken into consideration. Fig.8(a) shows the calculated cross-sectional temperature distribution of a PET substrate when it is at the highest temperature. The calculated temporal change in temperature at the position where the highest temperature occurs on the PET substrate is shown in Fig. 8 (b). The temperatures of the PET substrate seem to be above $\mathrm{Tg}$ within a PET substrate depth of about $0.8 \mu \mathrm{m}$ and reach $\mathrm{Tm}$ within a nearly $0.1 \mu \mathrm{m}$ depth.

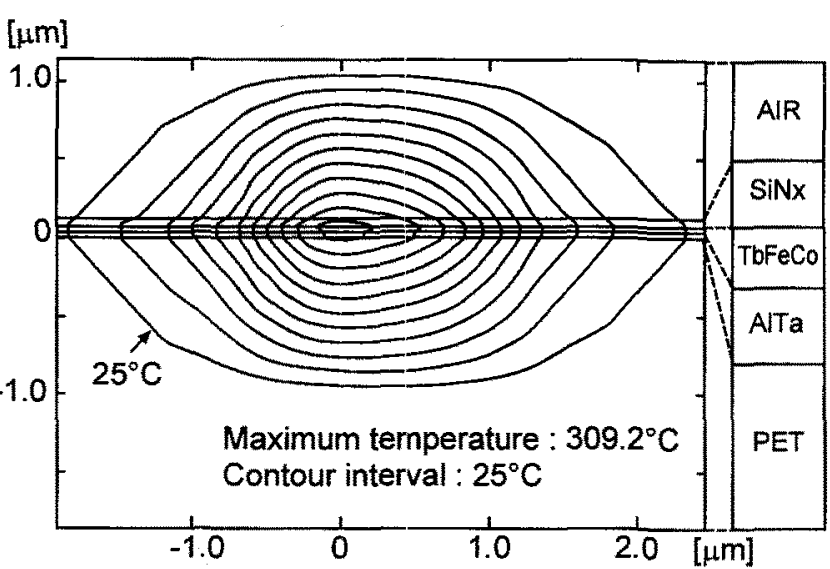

(a)

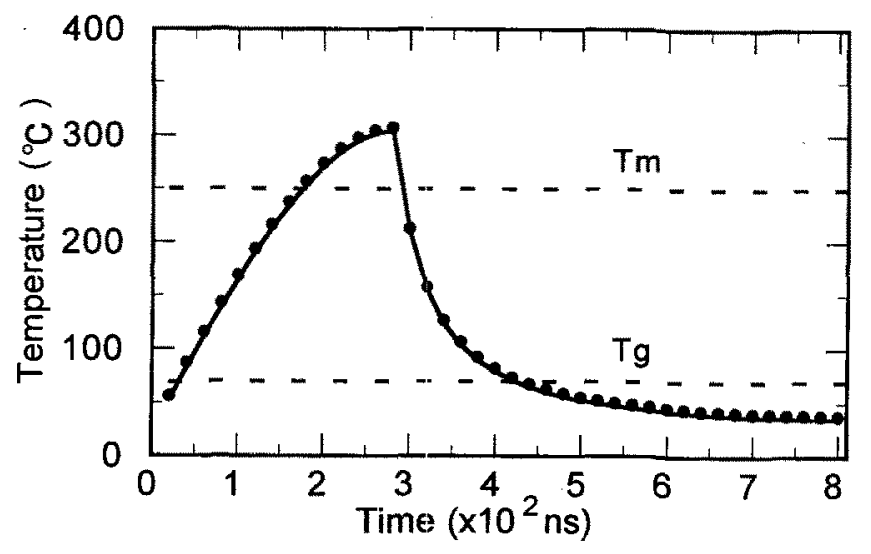

(b)

Fig.8. (a) Cross-sectional temperature distribution of PET substrate. (b) Temporary change in temperature at the position where the highest temperature occurs.

To investigate the effect of temperature rise, continuous erasing operations at a laser power of about $6 \mathrm{~mW}$, that was equal to the recording power, were carried out. CNR was measured every two thousand erasing operations. Figure 9(a) shows the results. No degradation in CNR was observed after ten thousand erasing operations. Figure 9 (b) shows the images observed with a polarizing microscope. The upper one is the track where ten thousand recording operations were carried out. And, the lower one is the track which was recorded once. Since both have almost the same track width, ten thousand erasing operations seem to have been done on the same track.

These results mean that ten thousand repetitions of a rise in temperature necessary for recording and erasing seems to cause no degradation in CNR in spite of partially melting the PET substrate. 


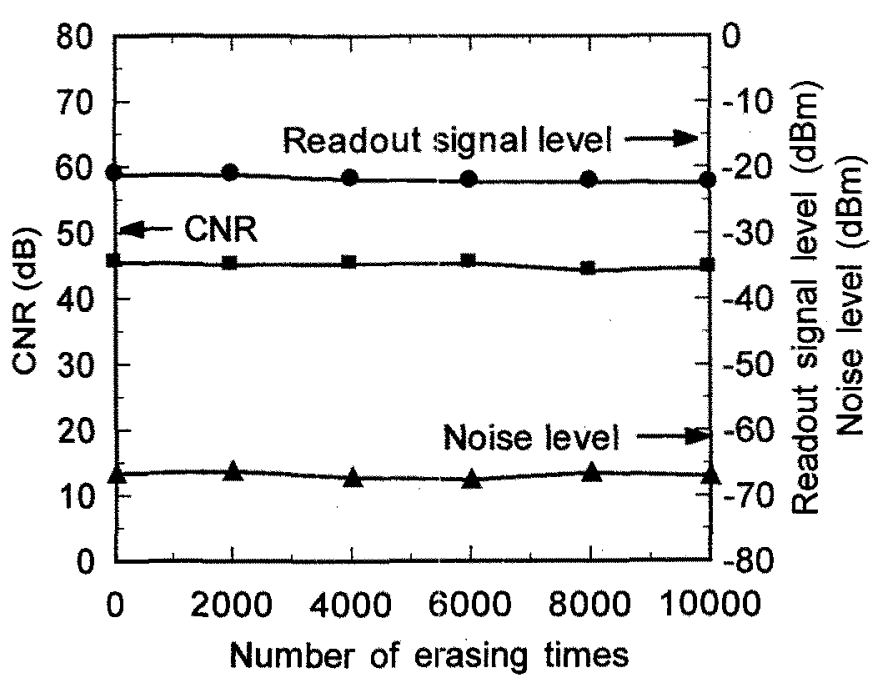

(a)

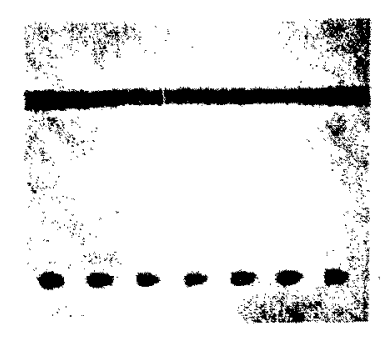

(b)

Fig.9. (a) CNR after ten thousand erasing operations. (b) Track images with a polarizing microscope.

\section{Tape tension and magnetic properties}

An MO tape is submitted to tension and deformation by bending under normal running and rewinding. Among these, we made experiments to investigate the effect of tension on magnetic and optical properties of a tape. A tri-layer (AITa: $45 \mathrm{~nm}$, TbFeCo: $28 \mathrm{~nm}$, SiNx: $600 \mathrm{~nm}$ ) was deposited on a PET substrate ( width: $1 / 2$ inch, length $600 \mathrm{~mm}$, thickness: $10 \mu \mathrm{m}) . R, \theta_{k}$ and coercive force $(\mathrm{HC}$ ) were measured under various tape tensions. $\mathrm{R}, \theta_{k}$ and $\mathrm{Hc}$ of the MO tape were respectively $16 \%, 1.15^{\circ}$ and $9.8 \mathrm{kOe}$ at a tape tension of $0.2 \mathrm{kgw}$.

Figure 10(a) shows the change of R. Stable measurement started beyond tape tension of $0.05 \mathrm{kgw}$ because the flatness necessary for exact measurement was accomplished for the first time under a tension of $0.05 \mathrm{kgw}$. The measured $\mathrm{R}$ was almost constant $(15 \% \sim 16 \%)$. The changes of $\theta_{k}$ and $\mathrm{Hc}$ are shown in Fig. $10(\mathrm{~b})$. While the $\mathrm{Hc}$ value decreased gradually from a tension of $0.5 \mathrm{kgw}, \theta_{k}$ and $\mathrm{R}$ held constant.

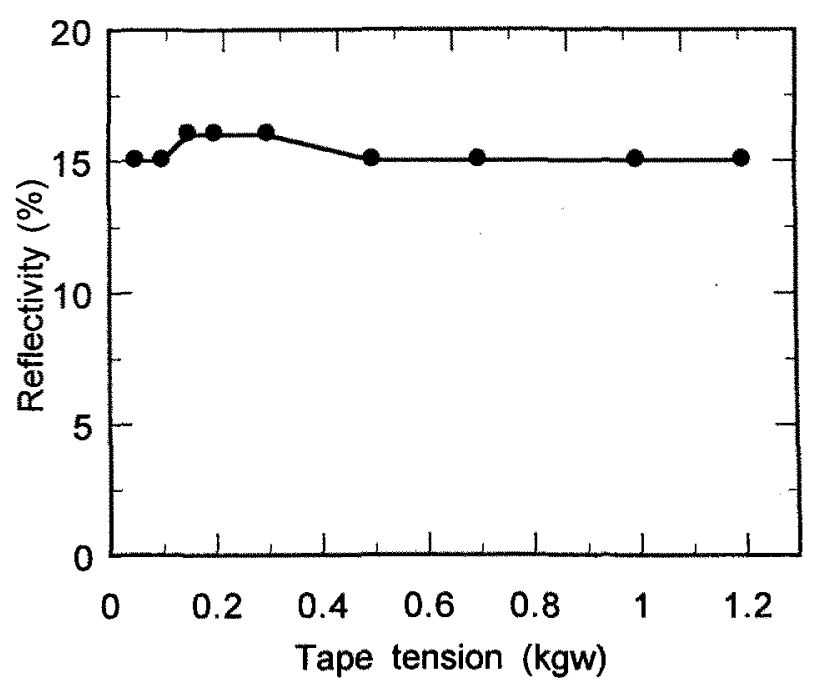

(a)

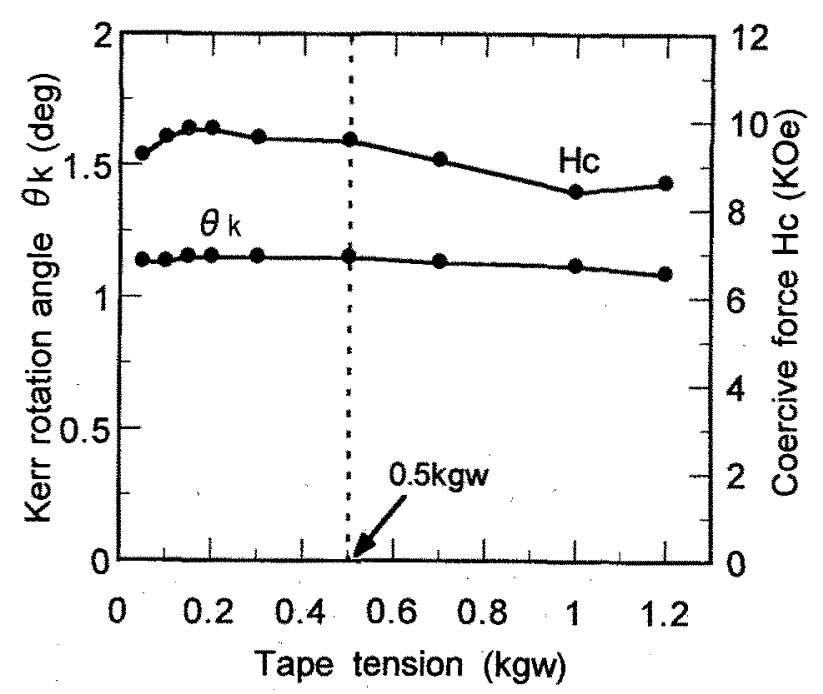

(b)

Fig.10. (a) Reflectivity vs tape tension. (b) $\theta k$ and $\mathrm{Hc}$ vs tape tension.

Therefore, it was found that the magnetic and optical properties of the MO tape medium were not degraded below a $0.5 \mathrm{kgw}$ tape tension.

Curling of MO tape

Deposition of three layers on a PET substrate causes significant curling of the tape. These layers have the stress shown in Table 3 and causes the curling in the same direction, that is, to make the trilayer side convex. Each layer was deposited on a Si(111) substrate ( diameter: $76 \mathrm{~mm}$, thickness: 188 $\mu \mathrm{m}$ ) for stress measurement. And, the stress of each 
Table 3. Stress of sputtered films.

\begin{tabular}{|c|c|}
\hline FILM & STRESS (dyne $\left./ \mathrm{mm}^{2}\right)$ \\
\hline AITa & $1.83 \times 10^{6}$ \\
TbFeCo & $4.41 \times 10^{7}$ \\
SiNx & $1.77 \times 10^{7}$ \\
\hline
\end{tabular}

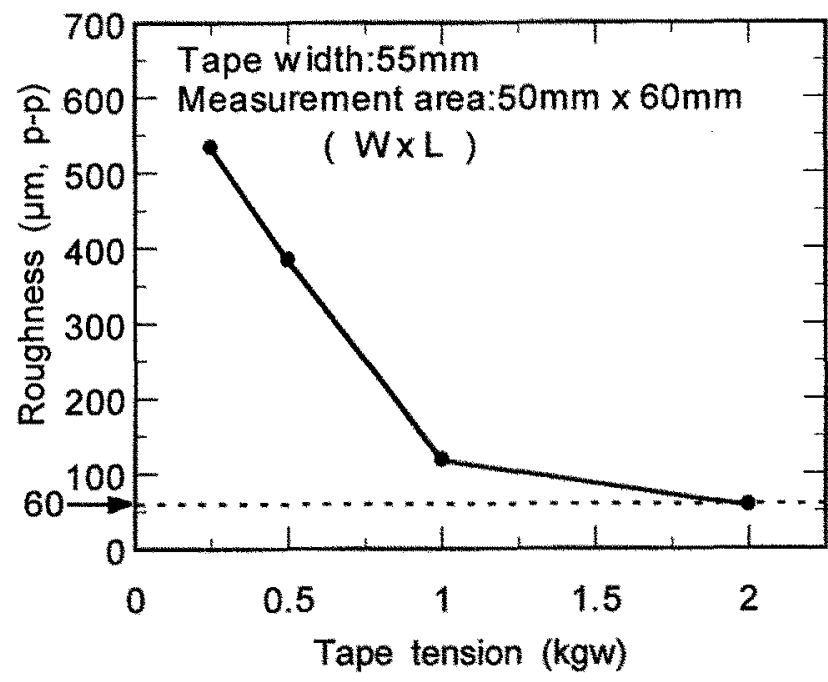

Fig.11. Relationship between tape tension and flatness.

layer was measured with a wafer deflection gauge ( stress gauge model 30114, IONIC SYSTEMS INC.). sufficient tape tension is necessary to satisfy the requirement for flatness of a MO tape. To measure the relationship between tape tension and flatness, the MO tape which was used for experiments with regard to surface roughness was flattened along two parallel rods located at an interval of $70 \mathrm{~mm}$.

Flatness ( $\mu \mathrm{m}$, peak to peak value ) of the scanning area (width: $50 \mathrm{~mm}$, length: $60 \mathrm{~mm}$ ) was measured with a laser displacement meter ( KL130B, ANRITSU Corp. ). And, tape tension was measured using a device which was made for this measurement. The measured results are shown in Fig.11. Tape tension of $2 \mathrm{kgw}$ made the MO tape flat below $60 \mu \mathrm{mp}-$ $p$. The flatness below $60 \mu \mathrm{mp}-\mathrm{p}$ needed sufficient tape tension beyond VTR tape tension. It is necessary to reduce further the curling of the tape.

\section{CONCLUSIONS}

We studied an MO tape theoretically and experimentally, and obtained the following results.

(1) In order to realize performances equal to an optical disk using a thin substrate,

- Incidence of laser is from the tri-layer side to avoid the birefringence of the PET substrate
- Surface roughness is below $\mathrm{Ra}=7 \mathrm{~nm}$

- Heat-resistance of PET substrate makes recording or erasing operations beyond ten thousands times possible

- MO tape ( width: $1 / 2$ inch, thickness: $10 \mu \mathrm{m}$ ) was stable in optical and magnetic properties under tape tension of $0.5 \mathrm{kgw}$

(2) A tri-layer structure is better for easy production

(3) Sufficient tape tension is necessary to satisfy the requirement for flatness since MO tape has a large extent of curling

\section{ACKNOWLEDGMENTS}

The authors wish to thank Professor T. Nomura of the Shizuoka Institute of Science \& Technology, Dr. I. Fujimoto and Dr. T. Tamaki for their useful advice and encouragement.

\section{REFERENCES}

[1] H. Tokumaru, K. Arai and S. Yoshimura: Tech. Dig. SOM'94 (1994) pp. 55-56

[2] M. Dancygier: IEEE Trans. on MAG. Vol. MAG-23, No.5 (1987) pp.2608-2610

[3] P. Vogelgesang and J. Hartmann: Proc. SPIE 899 (1988) pp.172-177

[4] H. Tokumaru, K. Arai, S. Yoshimura and H. Oshima: Jpn.J.Appl.Phys., Vol.32 (1993) pp.5428-5432

[5] H. Tokumaru, K. Arai and N. Kawamura: Jpn.J.Appl.Phys.,Vol.35 (1996) pp.375-379 